\title{
Avaliação do Conhecimento sobre Trauma Craniano Violento por Pais Brasileiros
}

\author{
Nahara Rodrigues Laterza Lopes ${ }^{1}$ \\ Lúcia Cavalcanti de Albuquerque Williams \\ Universidade Federal de São Carlos
}

\begin{abstract}
RESUMO - A ocorrência do trauma craniano violento (TCV) pode estar associada à falta de conhecimento de pais e cuidadores sobre o desenvolvimento infantil. Neste sentido, o presente estudo pretendeu investigar o conhecimento de pais sobre o choro do bebê, as estratégias para lidar com o choro e as consequências de sacudi-lo. Participaram da pesquisa 83 mães e 7 pais de recém-nascidos, que responderam ao instrumento Questionário sobre o Choro do Bebê. Os resultados do estudo sugerem um desconhecimento por parte dos pais sobre o TCV, principalmente no que se refere à sua gravidade. Além disso, 34,4\% dos participantes relataram que sacudiriam seus bebês para fazê-los parar de chorar. Tais resultados parecem indicar a necessidade de desenvolver intervenções de educação parental sobre o TCV, especialmente durante o pré-natal, de modo a contribuir para o aumento do conhecimento dos pais, prevenindo assim tal forma de maus-tratos.
\end{abstract}

Palavras-chave: trauma craniano violento, síndrome do bebê sacudido, abuso da criança, pais, relações pais-criança

\section{Brazilian Parents' Knowledge on Abusive Head Trauma}

\begin{abstract}
Abusive head trauma (AHT) may be associated to a lack of knowledge of parents and caregivers concerning child development. This study intended to investigate parental knowledge regarding the crying of babies, how parents deal with a crying baby and the consequences of shaking a baby to make it stop crying. Participants were 83 mothers and 7 fathers of newborns who responded to the Crying Baby Questionnaire. Results suggest a lack of knowledge of AHT, especially regarding its severity. Furthermore, $34.4 \%$ of participants reported that they would shake their babies to make them stop crying. These results highlight the need to develop interventions for parent training on AHT, particularly during the prenatal period in order to increase parental knowledge and to contribute to the prevention of this form of child maltreatment.
\end{abstract}

Keywords: abusive head trauma, shaken baby syndrome, child abuse, parents, parent child relations

O trauma craniano violento (TCV) é descrito pelo Centro de Controle e Prevenção de Doenças dos Estados Unidos como qualquer lesão craniana ou intracraniana decorrente de impacto brusco ou sacudida violenta em um bebê ou criança menor de cinco anos (Parks, Annest, Hill, \& Karch, 2012). Nesse sentido, a criança é submetida a severas e repetidas forças de aceleração e desaceleração, com ou sem o impacto direto da cabeça do bebê em alguma superfície, que resultam em um padrão característico de ferimentos, podendo incluir hemorragias na retina, fraturas, principalmente nas costelas e no final de ossos longos, assim como padrões reconhecidos de ferimentos cerebrais (Health Canada, 2001; National Center on Shaken Baby Syndrome, s.d.).

De acordo com Case, Graham, Handy, Jentzen e Monteleone (2001), entre as crianças que sofrem TCV, de 7 a 30\%, aproximadamente, morrem, de 30 a $50 \%$ apresentam déficits cognitivos e neurológicos significativos, e 30\% têm chance de recuperação total. Para o Centro de Controle de Doenças dos Estados Unidos (Center of Disease and Control, 2007), de 25 a $30 \%$ das crianças vítimas do TCV morrem e apenas $15 \%$ sobrevivem sem qualquer sequela, dado que aponta para a gravidade dessa forma de maus-tratos.

Uma característica relevante dessa forma de violência é o fato de o choro do bebê ser referido como o principal

1 Laboratório de Análise e Prevenção da Violência, Departamento de Psicologia - Universidade Federal de São Carlos, Rodovia Washington Luís, km 235, São Carlos, SP, Brasil. CEP: 13.565-905. E-mail: nahara. laterza@gmail.com gatilho para sua ocorrência (Case et al., 2001; Barr, Trent, \& Cross, 2006; Brooks \& Weathers, 2001; Commitee on Child Abuse and Neglect of the American Academy of Pediatrics, 2001; Hennes, Kini, \& Palusci, 2001; Lee, Barr, Catherine, $\&$ Wicks, 2007). Apesar de ser um importante meio de comunicação do bebê, o choro pode gerar sentimentos negativos nos cuidadores, tais como frustração, estresse e raiva, que podem servir de gatilho para ocorrência do TCV. De acordo com Leavitt (2001) a resposta parental ao choro do bebê é resultado da interação de três fatores: as características do próprio bebê, a cultura em que os pais estão inseridos e as crenças e expectativas que os pais apresentam sobre o bebê. Em relação ao primeiro fator, estudos apontam propriedades específicas do choro que contribuem para o sentimento de frustração que muitos cuidadores podem experienciar nos primeiros meses de vida de seu bebê (Barr, Paterson, MacMartin, Lehtonen, \& Young, 2005; Green \& Gustafson, 2001; James-Roberts, 2001). Essas propriedades podem ser resumidas por um aumento na média diária de duração do choro nas primeiras semanas de vida do bebê, que atinge um pico por volta do segundo mês ( $6^{\mathrm{a}}$ semana), apresentando uma redução após o pico. Após o quarto mês de vida, o choro se torna mais intencional e mais relacionado com os eventos ambientais (Brazelton, 1962). Cabe destacar que esse padrão é observado mesmo quando consideradas as variações individuais na quantidade de horas que um bebê pode chorar por dia (Barr, 2014). Ainda que o número médio diário de horas de choro do bebê não esteja bem estabelecido na literatura, é esperado que o bebê chore por volta de duas a 
duas horas e meia por dia (James-Roberts, 2001). O padrão de choro do bebê nos primeiros meses de vida inclui ainda episódios de choro prolongado, inconsolável e imprevisível, que ocorrem normalmente no final da tarde ou início da noite e são acompanhados por expressões faciais de dor e aumento do tônus muscular (Barr et al., 2006; Lee et al., 2007).

Esse padrão de choro, denominado de curva normal do choro, foi observado não apenas em bebês humanos ocidentais, como também em diferentes populações, tais como em bebês !Kung San, uma sociedade do noroeste de Botswana, bebês pré-termo e, inclusive, em bebês mamíferos não primatas (Alvarez \& James-Roberts, 1996; Barr, Chen, Hopkins, \& Westra, 1996; Barr, Konner, Bakeman, \& Adamson, 1991; Hofer, 2001; Hunziker \& Barr, 1986; JamesRoberts \& Halil, 1991; Kramer et al., 2001; Pettijohn, 1979; Wessel, Cobb, Jackson, Harris, \& Detwiler, 1954). Apesar do aparente caráter universal desse padrão, diferentes culturas parecem se distinguir no modo como cuidam de seus bebês e como atribuem significados ao seu choro (Leavitt, 2001). Por exemplo, na comunidade !Kung San o padrão de cuidados observado é caracterizado por um contato constante da mãe com o bebê, uma amamentação frequente (aproximadamente quatro vezes por hora), curta (duração de um a dois minutos cada) e prolongada (até o segundo ou terceiro ano de vida do bebê), bem como pela manutenção do bebê em uma postura ereta durante a maior parte do tempo, devido ao uso do carregador de bebê (sling) (Barr, 2001; Barr et al., 1991). Tal padrão é bastante contrastante com o observado na cultura norte-americana, em que o contato com o bebê não é tão frequente, a amamentação ocorre de forma mais regular e controlada e o bebê permanece a maior parte do tempo deitado. Da mesma forma, a percepção sobre o significado do choro pode variar muito entre culturas. No Japão, por exemplo, o choro do bebê é um indicativo de força e saúde (Fujiwara et al., 2012), enquanto nos Estados Unidos ele é frequentemente associado à ocorrência da cólica, e, portanto, a um problema de saúde no bebê (Barr, 2014).

Como apontado por Leavitt (2001), essa percepção parental sobre o choro, influenciada pela cultura e pelas suas próprias experiências de vida, exerce um papel importante na resposta emitida pelos pais diante do choro do bebê, podendo ser mais relevante para a ocorrência dos maus-tratos do que as propriedades do choro em si (Reijneveld, Wal, Brugmanm, Sing, \& Verloove-Vanhorick, 2004). De acordo com Dix e Reinhold (1991), atribuições negativas do choro podem levar à hostilidade, agressão e a respostas centradas no bem-estar dos pais e não do bebê.

Dada a especificidade do mecanismo de lesão do TCV, bem como o fato de o choro do bebê ser seu principal fator de risco, diversos programas têm sido desenvolvidos e implementados exclusivamente para a prevenção dessa forma de maus-tratos (Keenan \& Leventhal, 2010; Runyan et al., 2009; Tolliday et al., 2010, Dias et al., 2005; Deyo, Skybo, \& Carroll, 2008). Desenvolvidos especialmente no Canadá, Estados Unidos e Austrália, esses programas objetivam fornecer informações sobre o padrão normal do choro do bebê e os riscos de sacudi-lo, bem como ensinar a pais e cuidadores técnicas para lidar de forma saudável com seu bebê, principalmente quando ele chora (Deyo et al., 2008;
Dias et al., 2005; Frasier, 2008; Keenan \& Leventhal, 2010; Runyan et al., 2009; Tolliday et al., 2010).

Após revisão nas bases de dados MEDLINE, SciELO, PsycINFO e LILACS com as palavras-chave "síndrome do bebê sacudido" e "trauma craniano violento" no ano de 2012, foi encontrado apenas um estudo empírico no Brasil sobre essa temática, realizado por Fernandes, Silva e Javorski (2010). As autoras investigaram o conhecimento de médicos, enfermeiros, técnicos de enfermagem e agentes comunitários de saúde do Programa de Saúde da Família de um município do interior de Pernambuco a respeito dessa forma de maustratos, tendo encontrado que $97 \%$ desses profissionais não conheciam o TCV. Uma das principais implicações desse desconhecimento é a impossibilidade de identificação dos casos e, consequentemente, de sua prevenção (Fernandes et al., 2010).

Tendo em vista a escassez de estudos nacionais sobre o TCV e a importância da atribuição parental sobre o choro do bebê para a ocorrência dessa forma de maus-tratos, o presente artigo teve como objetivo investigar a percepção de pais sobre o choro do bebê nas primeiras semanas de vida, bem como as principais estratégias para lidar com o choro e a compreensão das consequências de se sacudir um bebê.

\section{Método}

\section{Participantes}

Participaram do estudo 82 mães, 07 pais de recémnascidos e 01 gestante, totalizando 90 participantes. Todos os pais ${ }^{1}$ se encontravam na maternidade por ocasião do nascimento dos filhos. A idade dos participantes variou de 14 a 42 anos, sendo que a média foi de 26 anos. Em relação à idade dos 90 bebês, esta variou de 0 a 3 dias, sendo que $90 \%$ deles tinham apenas um dia de vida.

A escolaridade dos participantes variou de Ensino Fundamental Incompleto a Ensino Superior Completo, sendo que 32\% apresentavam Ensino Médio Completo e $21 \%$ Ensino Médio Incompleto. Em relação à condição socioeconômica, 49\% dos participantes classificaram-se na classe $\mathrm{C}$, que corresponde a uma renda familiar média entre $\mathrm{R} \$ 962,00$ e R\$1.459,00. Oitenta por cento dos participantes afirmaram ter tido experiência prévia no cuidado de bebês, sendo que desses $91 \%$ afirmaram uma frequência de cuidado de cinco dias por semana ou mais. A coleta de dados foi realizada na maternidade de um município de médio porte do interior do Estado de São Paulo (221.950 habitantes) ${ }^{2}$.

\section{Instrumentos}

Após revisão da literatura nacional e internacional em busca de instrumentos que avaliassem o conhecimento de pais

1 Ao se utilizar o termo pais, os autores estão se referindo tanto a pais como a mães

2 Fonte de Dados: Contagem da População de 2010 pelo Instituto Brasileiro de Geografia e Estatística (IBGE). 
sobre o TCV, foram encontrados apenas dois instrumentos publicados, sendo eles o Shaken Baby Syndrome Awareness Assessment - Short Version (Russell, 2010) e o Crying Baby Questionnaire (Tolliday et al., 2010). Dado que o questionário de Russell (2010) objetiva avaliar apenas as atitudes de pais frente ao choro do bebê, optou-se por utilizar o Crying Baby Questionnaire. Desenvolvido por Tolliday et al. (2010), na Austrália, para a avaliação do programa de prevenção Shaken Baby Prevention Project (Projeto de Prevenção da Síndrome do Bebê Sacudido) e implementado no The Children's Hospital at Westmead (Hospital da Criança de Westmead), esse instrumento tem como principal objetivo avaliar o conhecimento de pais sobre os possíveis motivos para o choro do bebê, as estratégias para lidar com o choro do bebê, as consequências de sacudi-lo, o número médio de horas de choro esperado para o bebê e os sentimentos gerados pelo cuidado do bebê.

Em sua versão original, o instrumento apresenta (a) uma questão múltipla-escolha em que o respondente deve indicar se ele acredita que os motivos expostos podem fazer o bebê chorar a partir de uma escala tipo Likert de três pontos (Sim, Não ou Não Sei), (b) uma questão aberta em que o respondente deveria indicar o que ele poderia fazer diante do choro do bebê, (c) uma questão fechada em que o respondente deve indicar seu grau de concordância com a afirmação "Cuidar de um bebê pode ser frustrante e estressante", (d) uma questão múltipla-escolha em que o respondente deve indicar o número médio de horas que um bebê saudável pode chorar por dia e (e) uma questão aberta em que o respondente deveria indicar quais as possíveis consequências de se sacudir um bebê (Tolliday et al., 2010).

Para utilização no Brasil, o instrumento (Tolliday et al., 2010) foi traduzido e adaptado após autorização dos autores. Esse processo iniciou-se com a tradução literal de cada questão pela primeira autora, que foi revisada pela segunda. Em seguida, cada item foi avaliado quanto à gramática, semântica, sintaxe e pertinência. O questionário foi, então, enviado a três juízes, sendo um pesquisador da área de violência contra a criança e dois da área de desenvolvimento infantil, para que os mesmos analisassem a representatividade e a clareza dos itens. Cada juiz recebeu uma carta solicitando a colaboração na avaliação do instrumento, uma cópia do instrumento e um material escrito apresentando a descrição e especificidade teórica do material. As sugestões de cada juiz foram avaliadas e, quando consideradas pertinentes, incluídas no instrumento.

Além disso, foi realizado um estudo piloto com 52 estudantes universitários e profissionais da área da saúde e educação, por ocasião de um seminário sobre o TCV. O questionário foi aplicado antes e após a realização de uma palestra sobre o TCV e a exibição do vídeo "Lidando com o Choro do Bebê", desenvolvido pela mesma equipe australiana do programa The Shaken Baby Prevention Project para utilização em suas ações preventivas, e traduzido e adaptado para o português pelo Laboratório de Análise e Prevenção da Violência da Universidade Federal de São Carlos, pelo Centro de Estudos Integrados Infância, Adolescência e Saúde (CEIIAS), pelo Instituto Zero a Seis e pelo Grupo Especial de Interesse em Saúde da Criança e do Adolescente da Rede Universitária de Telemedicina (RUTE).
A avaliação dos resultados mostrou que, após a intervenção, os participantes passaram a atribuir a razão do choro menos a características do bebê e mais a fatores ambientais, bem como a afirmar de forma mais adequada a média diária de horas que o bebê costuma chorar, demonstrando, assim, a viabilidade do instrumento para o presente estudo. Apesar disso, o instrumento apresentava algumas limitações que dificultavam a análise dos dados obtidos, como, por exemplo, a presença de escalas tipo Likert com apenas 3 pontos. Além disso, o fato de as questões sobre as estratégias para lidar com o choro do bebê e as consequências de sacudi-lo serem abertas possibilitou a ocorrência de respostas evasivas que não mediam o conhecimento dos participantes sobre os temas.

Dadas essas limitações, foram realizadas algumas modificações no instrumento de modo a torná-lo mais objetivo. As escalas tipo Likert de três pontos (Sim, Não ou Não Sei) foram substituídas por escalas Likert de cinco pontos (Discordo Fortemente, Discordo, Não Sei, Concordo e Concordo Fortemente), permitindo maior variabilidade das respostas. Além disso, as respostas oferecidas pelos participantes no estudo piloto para as questões abertas foram transformadas em itens que descreviam possíveis estratégias a serem utilizadas diante do choro do bebê ou possíveis consequências de se sacudir um bebê. Assim, na nova versão do instrumento, o participante deveria avaliar seu grau de concordância para cada um desses itens utilizando uma escala tipo Likert de cinco pontos.

Em sua versão final, o Questionário sobre o Choro do Bebê aborda cinco aspectos relacionados ao TCV: (a) o conhecimento dos pais sobre as possíveis razões para o choro do bebê (20 itens), (b) o conhecimento dos pais sobre as estratégias para lidar com o choro do bebê (23 itens), (c) o conhecimento dos pais sobre as consequências de se sacudir um bebê (15 itens), (d) o conhecimento dos pais sobre o número médio de horas que um bebê saudável pode chorar por dia (1 item), e (e) os sentimentos associados ao cuidado do bebê (1 item). A Tabela 1 apresenta as questões de cada aspecto do instrumento, bem como um exemplo de item e suas opções de respostas.

\section{Procedimento}

Comitê de ética. A maternidade foi contatada para que autorizasse a realização da coleta de dados em suas dependências. Após autorização, o projeto foi enviado ao Comitê de Ética em Pesquisa em Seres Humanos da Universidade Federal de São Carlos, que emitiu parecer favorável à realização da pesquisa (Parecer no 338/2011).

Coleta de dados. Após aprovação da pesquisa pelo Comitê de Ética, iniciou-se a coleta de dados na maternidade. Os nomes e os leitos das possíveis participantes eram fornecidos pelas enfermeiras do serviço de Banco de Leite da maternidade. Essas profissionais realizavam um levantamento diário de todas as mulheres que haviam tido filho no dia anterior para que pudessem passar as orientações sobre aleitamento. Tal levantamento era passado para a primeira autora, que realizava o sorteio de duas mães para realizar a intervenção. 
A primeira autora se dirigia até o leito da puérpera sorteada, convidando-a a participar da pesquisa. Em caso de recusa ou desistência, era sorteada outra mãe. Na maternidade onde ocorreu a coleta de dados, havia três possibilidades de configuração dos quartos: (a) quatro leitos e um banheiro compartilhado; (b) dois leitos e um banheiro compartilhado com outro quarto com dois leitos; e (c) um leito com banheiro privado. Assim, quando as participantes eram internadas em quartos com mais de um leito, todas as outras mães internadas no mesmo quarto eram também convidadas a participar da pesquisa.

No leito de cada participante, a primeira autora explicava os objetivos, o procedimento, os riscos e benefícios da pesquisa, bem como garantia seu anonimato. Quando o parceiro da puérpera estava presente no quarto, ele também era convidado a participar do estudo. Era, então, solicitado o consentimento referente à participação de ambos por meio da assinatura do Termo de Consentimento Livre e Esclarecido (TLCE). Cada participante deveria assinar o seu próprio TCLE, ou seja, um para a puérpera e outro para o parceiro.

Em seguida o Questionário sobre o Choro do Bebê era respondido por meio de entrevista, ou seja, a primeira autora lia os itens e anotava as respostas dos participantes. A escolha por realizar a aplicação do questionário por meio de entrevista ocorreu devido a uma questão prática, uma vez que as mães frequentemente estavam com seus bebês no colo. Nas ocasiões em que o parceiro também aceitava participar do estudo, era solicitado que ele respondesse o questionário individualmente, ao mesmo tempo em que a primeira autora aplicava o instrumento com a mãe, de modo a reduzir o tempo gasto pelos participantes com o estudo. A coleta de dados ocorreu ao longo de oito meses.

\section{Análise dos Dados}

Por se tratar de uma pesquisa de levantamento (Cozby, 2011), optou-se por utilizar estatística descritiva, especialmente a frequência de repostas, para análise dos dados. Para o cálculo das frequências, foi utilizado o software estatístico SPSS versão 20.

Os itens das questões sobre as possíveis razões para o choro do bebê, as possíveis estratégias para lidar com o choro e as consequências de sacudir um bebê foram agrupados de acordo com seu conteúdo, de modo a simplificar a análise de dados. Desse modo, os itens sobre a percepção dos pais acerca das possíveis razões para o choro foram agrupados em quatro categorias: (a) razões relacionadas às condições do bebê, ou seja, itens que descrevem que o choro é causado

Tabela 1 Exemplos de questão, item e opções de respostas para cada aspecto do instrumento Questionário sobre o Choro do Bebê

\begin{tabular}{|c|c|c|c|c|c|c|c|c|}
\hline \multirow{2}{*}{ 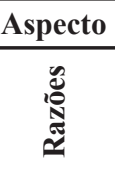 } & \multirow{2}{*}{\begin{tabular}{l}
\multicolumn{1}{c}{ Questão } \\
O que você acha que \\
pode fazer um bebê \\
chorar?
\end{tabular}} & \multirow{2}{*}{\begin{tabular}{l}
\multicolumn{1}{c}{ Item } \\
$\begin{array}{l}\text { O bebê estar } \\
\text { doente. }\end{array}$
\end{tabular}} & \multicolumn{6}{|c|}{ Opções de Resposta } \\
\hline & & & $\begin{array}{l}\text { Discordo } \\
\text { Fortemente }\end{array}$ & Discordo & Não Sei & Conco & & $\begin{array}{l}\text { Concordo } \\
\text { Totalmente }\end{array}$ \\
\hline 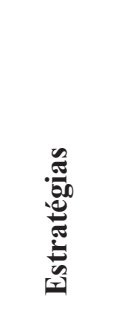 & $\begin{array}{l}\text { Imagine que você está } \\
\text { sozinho, cuidando de } \\
\text { um bebê e este não pára } \\
\text { de chorar. Atribua uma } \\
\text { nota de } 0 \text { a } 5 \text { para cada } \\
\text { alternativa abaixo de } \\
\text { acordo com o que você } \\
\text { faria nesta situação. }\end{array}$ & $\begin{array}{l}\text { Alimentaria } \\
\text { o bebê } \\
\text { novamente. }\end{array}$ & Nunca Faria & $\begin{array}{l}\text { Provavelmente } \\
\text { Não Faria }\end{array}$ & Não Sei & $\begin{array}{r}\text { Provavel } \\
\text { Fari }\end{array}$ & nente & $\begin{array}{l}\text { Certamente } \\
\text { Faria }\end{array}$ \\
\hline 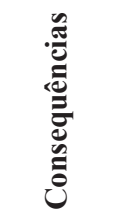 & $\begin{array}{l}\text { O que você acredita que } \\
\text { pode acontecer com o } \\
\text { bebê se ele for sacudido? }\end{array}$ & $\begin{array}{l}\text { O bebê pode } \\
\text { vomitar. }\end{array}$ & & Sim & Não & & Não & \\
\hline : & $\begin{array}{l}\text { Cuidar de um bebê pode s } \\
\text { estressante. }\end{array}$ & er cansativo e & $\begin{array}{l}\text { Discordo } \\
\text { Fortemente }\end{array}$ & Discordo & Não Sei & Concordo & & $\begin{array}{l}\text { Concordo } \\
\text { Totalmente }\end{array}$ \\
\hline 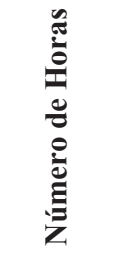 & $\begin{array}{l}\text { Quantas horas por dia um } \\
\text { saudável pode chorar? }\end{array}$ & bebê normal e & 0 a 1 & 2 a 3 & & 4 a 5 & & 6 ou mais \\
\hline
\end{tabular}


por seu estado físico, tais como estar com fome, com dor ou doente (6 itens); (b) razões relacionadas às características de personalidade do bebê, ou seja, itens que descrevem que o choro do bebê ocorre devido a seus traços pessoais, tais como o bebê ser "mimado" ou impaciente (10 itens); (c) razões relacionadas ao cuidador ou ao ambiente, ou seja, itens que descrevem motivos para o choro do bebê relacionados aos estados do cuidador, como estar estressado, e do ambiente, como estar barulhento ( 2 itens); e (d) padrão de choro do bebê, cujos itens não se referem a possíveis razões para o choro, mas abordam o conhecimento do cuidador sobre o seu padrão nos primeiros meses de vida (2 itens).

As alternativas de estratégias para lidar com o choro do bebê oferecidas pelo instrumento foram classificadas em cinco categorias, a saber: (a) estratégias relacionadas à satisfação das necessidades básicas do bebê (3 itens), (b) estratégias relacionadas ao cuidador, ou seja, estratégias que o cuidador pode utilizar para regular suas próprias emoções (6 itens); (c) estratégias relacionadas ao ambiente do bebê, tais como colocá-lo em um lugar mais escuro (3 itens); (d) estratégias negativas, como, por exemplo, gritar com o bebê (3 itens); e (e) estratégias relacionadas ao bebê, ou seja, aquelas direcionadas ao bebê, mas que não são usadas para a satisfação de suas necessidades básicas, como, por exemplo, fazer uma massagem no bebê ( 8 itens). Para a análise dos dados obtidos por meio dessas questões, as respostas Discordo Totalmente e Discordo foram agrupadas na categoria Discordo. Da mesma forma, as respostas Concordo Totalmente e Concordo foram agrupadas na categoria Concordo. Por fim, os itens referentes às consequências de se sacudir um bebê foram classificados em três categorias: (a) consequências graves, tais como a morte do bebê ( 9 itens); (b) consequências não graves, tais como o bebê vomitar (4 itens); e (c) consequências inadequadas, ou seja, itens que não correspondem a consequências reais de se sacudir um bebê, tais como o bebê se acalmar (2 itens).

\section{Resultados e Discussão}

As análises descritivas serão apresentadas de acordo com cada aspecto medido pelo instrumento: (a) o conhecimento sobre as possíveis razões para o choro do bebê; (b) o conhecimento sobre as estratégias para lidar com o choro do bebê; (c) o conhecimento sobre as consequências de sacudir o bebê; (d) o conhecimento sobre o número médio diário de horas que um bebê pode chorar; e (e) os sentimentos associados ao cuidado do bebê.

\section{Possíveis Razões para o Choro do Bebê}

A Tabela 2 apresenta as porcentagens de resposta para cada item do questionário relativo às razões do choro do bebê, separados por categoria. Como pode ser observado na tabela, os participantes concordaram mais com motivos relacionados às condições do bebê $(84,1 \%)$, do que com aqueles relacionados a suas características de personalidade $(55,5 \%)$ ou às condições do cuidador ou do ambiente $(78,9 \%)$. Entre as razões apontadas como menos prováveis de fazer o bebê chorar, estão aquelas relacionadas às características do bebê (29\%), principalmente o bebê ser exigente e malcomportado, com $41,1 \%$ e $40 \%$ de discordância, respectivamente. Em suma, apesar de os participantes atribuírem com frequência

Tabela 2 Porcentagens de resposta para cada item do questionário relativo às razões do choro do bebê $(n=90)$

\begin{tabular}{|c|c|c|c|}
\hline & $\%$ Concordo & \% Discordo & $\begin{array}{c}\% \\
\text { Não Sei }\end{array}$ \\
\hline $\begin{array}{l}\text { Características de } \\
\text { Personalidade do Bebê }\end{array}$ & 55,5 & 29 & 14,7 \\
\hline Bebê é mimado & 76,7 & 13,3 & 10,0 \\
\hline Bebê é mal comportado & 41,1 & 40,0 & 18,9 \\
\hline Bebê é genioso & 53,3 & 31,1 & 15,6 \\
\hline Bebê é muito exigente & 37,8 & 41,1 & 21,1 \\
\hline Bebê é teimoso & 50,0 & 35,6 & 14,4 \\
\hline Bebê é impaciente & 76,7 & 14,4 & 8,9 \\
\hline Condições do Bebê & 84,1 & 10,4 & 5,4 \\
\hline Bebê estar doente & 94,4 & 3,3 & 2,2 \\
\hline Bebê estar cansado & 64,4 & 26,7 & 8,9 \\
\hline Bebê estar com fome & 99,0 & 0,0 & 1,0 \\
\hline Bebê estar com fralda suja & 95,6 & 4,4 & 0,0 \\
\hline Bebê estar com dor & 99,0 & 0,0 & 1,0 \\
\hline $\begin{array}{l}\text { Leite da mamadeira está } \\
\text { ruim }\end{array}$ & 83,3 & 8,9 & 7,8 \\
\hline Bebê estar entediado & 62,2 & 23,3 & 14,4 \\
\hline Bebê estar triste & 56,7 & 28,9 & 14,4 \\
\hline $\begin{array}{l}\text { Bebê estar reclamando } \\
\text { de algo }\end{array}$ & 93,3 & 3,3 & 3,3 \\
\hline Bebê querer colo & 93,3 & 4,4 & 2,2 \\
\hline $\begin{array}{l}\text { Condições do Cuidador } \\
\text { ou do Ambiente }\end{array}$ & 78,9 & 11,1 & 10 \\
\hline Cuidador está estressado & 74,4 & 12,2 & 13,3 \\
\hline Ambiente está barulhento & 83,3 & 10,0 & 6,7 \\
\hline $\begin{array}{l}\text { Padrão de Choro do } \\
\text { Bebê }\end{array}$ & 49,4 & 4,4 & 46,1 \\
\hline $\begin{array}{l}\text { É natural que um bebê } \\
\text { chore }\end{array}$ & 80 & 2,2 & 17,8 \\
\hline $\begin{array}{l}\text { Os bebês choram sem } \\
\text { motivo }\end{array}$ & 18,9 & 6,7 & 74,4 \\
\hline
\end{tabular}

as razões do choro a condições do bebê, eles também consideraram que as características de personalidade do bebê podem contribuir para este comportamento.

Entende-se traço de personalidade como uma característica relativamente estável na forma de pensar, sentir e se comportar (Sisto, 2004), sendo, nesse sentido, difícil de ser modificada e podendo permanecer com o bebê por um longo período ou até o final de sua vida. De acordo com Nash, Morris e Goodman (2008), considerar a atribuição feita pelos pais ou cuidadores sobre as possíveis razões do choro é importante, visto que essa percepção poderá influenciar a escolha das estratégias a serem utilizadas no cuidado do bebê. Ao perceber o choro como decorrente de uma característica imutável do bebê, como ser "mimado" ou "impaciente", implica-se uma ideia de que nada pode 
ser feito para mudar o comportamento do indivíduo, nesse caso, o choro do bebê. Essa atribuição negativa do choro pode levar à hostilidade, à agressão e a respostas centradas na satisfação das necessidades do cuidador e não no bebê (Tolliday et al., 2010).

Atribuir, por outro lado, as causas do choro a fatores externos ao bebê, como, por exemplo, ao seu estado atual ou à condição do ambiente, aumenta o número de estratégias disponíveis para o cuidador lidar com o choro, reduzindo a possibilidade do aparecimento de sentimentos de frustração ou raiva e a probabilidade de o cuidador sacudir seu bebê. Nesse sentido, ressalta-se a importância de intervenções educacionais, especialmente durante o pré-natal, que possam alterar as crenças dos cuidadores sobre as possíveis causas do choro do bebê, contribuindo para a prevenção do TCV.

Em relação ao padrão de choro do bebê, $80 \%$ dos participantes concordaram ser natural um bebê chorar e $74,4 \%$ discordaram que o choro do bebê poderia ocorrer sem qualquer razão. Esses dados indicam um bom conhecimento dos participantes sobre o padrão de choro do bebê. Cabe destacar, entretanto, que o item "Bebês choram sem motivo" deveria ser alterado para "Bebês choram sem uma razão aparente", de modo a se aproximar com o proposto na literatura da área, que aponta a ocorrência de episódios de choro inconsolável e sem razão aparente (Barr et al., 2005; Barr et al., 2006; Lee et al., 2007).

Nash et al. (2008) investigaram a opinião de 24 mães e dois pais sobre os sentimentos evocados pelo choro do bebê, os fatores que influenciam o modo como lidam com o choro do bebê e as possíveis razões para o choro. Os pais entrevistados relataram que um dos principais motivos para o choro do bebê era ganhar atenção, obtendo colo.

O presente estudo parece corroborar esses dados, uma vez que $93,3 \%$ dos participantes concordaram que o bebê querer colo pode ser um possível motivo para o choro. De acordo com Nash et al. (2008), essa percepção de intencionalidade do comportamento do bebê pode decorrer de uma interpretação inadequada dos pais sobre o fato de os bebês se acalmarem após ganharem a atenção dos pais em resposta ao seu choro. Esses autores ressaltam, entretanto, que é preciso cautela com essa percepção dos pais acerca do choro do bebê, visto que, em alguns casos, acreditar que um bebê chore apenas para ganhar atenção pode gerar sentimentos de ressentimento e frustração, aumentando o risco para ocorrência de maustratos, especialmente o TCV.

\section{Estratégias para Lidar com o Choro do Bebê}

A Tabela 3 apresenta as porcentagens de resposta para cada item do questionário relativo às estratégias para lidar com o choro do bebê, separados por categoria. Entre as principais estratégias utilizadas pelos participantes, encontram-se aquelas relacionadas ao bebê $(88,6 \%)$ e à satisfação de suas necessidades básicas $(88,5 \%)$. Esses dados corroboram os encontrados no estudo australiano de Tolliday et al. (2010), em que 116 cuidadores responderam ao Crying Baby Questionnaire. No estudo, 84\% dos participantes relataram que utilizariam estratégias relacionadas diretamente ao bebê para lidar com seu choro.

Em contraste, as estratégias menos prováveis de serem utilizadas foram as negativas ( $84,4 \%$ de discordância), como, por exemplo, gritar com o bebê, e as relacionadas ao cuidador (53,3\% de discordância). Esses resultados parecem reforçar a hipótese de que as crenças sobre as razões do choro do bebê influenciam a escolha das estratégias para lidar com ele. $\mathrm{O}$ fato de que $55,5 \%$ dos participantes atribuíram ao choro razões relacionadas à personalidade do bebê pode oferecer uma das explicações para a alta discordância quanto às estratégias relacionadas ao cuidador.

Outra explicação para essa discordância pode estar relacionada a uma possível crença de que, quando o bebê chora, o cuidador deve manter-se próximo a ele. Entre as estratégias relacionadas ao bebê mais utilizadas, estão "Trazer o bebê para perto do corpo" (100\%) e "Abraçar o bebê" (95,6\%). Em contrapartida, as estratégias menos utilizadas relacionadas ao cuidador foram: "Deixar o bebê sozinho e sair do quarto por um tempo para assistir televisão ou ouvir uma música" (2,2\%), "Deixar o bebê sozinho e sair do quarto por um tempo para respirar fundo e se acalmar" (10\%) e "Colocar o bebê no berço" $(24,4 \%)$.

A proximidade ao bebê, nessas situações, é adequada e recomendada para o fortalecimento do vínculo cuidadorbebê (Lopez \& Júnior, 2010). Entretanto, essas estratégias são adequadas somente quando os cuidadores encontram-se calmos e pacientes. Quando o choro gera sentimentos de frustração, incapacidade, raiva ou desespero, a estratégia mais adequada para a situação consiste em afastar-se do ruído do choro, deixando o bebê sozinho em um lugar seguro ou, quando possível, na companhia de alguém mais experiente e calmo (Runyan \& Zolotor, 2009). Nesse sentido, é importante ensinar os pais a identificarem seus próprios sentimentos, especialmente a raiva, o estresse e a frustração, assim como a lidarem de forma adequada com esses sentimentos e, principalmente a identificarem os tipos de estratégias que podem utilizar nesses momentos para lidar de forma segura com o bebê choroso (Runyan \& Zolotor, 2009).

De acordo com Nash et al. (2008), parece haver uma diferença de gênero em relação à estratégia de pedir ajuda para lidar com o bebê. Em seu estudo, metade dos cuidadores entrevistados relatou que, diante do choro do filho, os pais (homens) passavam o cuidado para a mãe ou avó do bebê (Nash et al., 2008). No presente estudo, as mães concordaram mais em pedir ajuda $(62,7 \%$ de concordância para o item referente a pedir ajuda para alguém mais experiente e 55,4\% para o item referente a pedir ajuda para alguém mais calmo) do que os pais (42,9\% para ambos os itens). Entretanto, ressalta-se a necessidade de mais estudos que investiguem essa questão, visto que o número reduzido de homens na amostra impossibilita conclusões definitivas.

Apesar de os participantes discordarem da utilização das estratégias relacionadas ao cuidador para lidar com o choro do bebê, estas foram mais prováveis de serem utilizadas no presente estudo do que no de Tolliday et al. (2010), em que apenas $11 \%$ dos participantes consideraram utilizá-las. Além disso, os participantes do presente estudo mostraramse mais favoráveis à utilização das estratégias relacionadas 
ao ambiente do bebê $(62,6 \%)$ comparando-se com a amostra australiana (5\%). Esses dados parecem indicar uma diferença cultural em relação às estratégias utilizadas pelos cuidadores para lidar com o bebê, ressaltando a necessidade de estudos nacionais sobre o tema.

Em relação ao item "Sacudiria o bebê para tentar acalmá1o", 34\% dos participantes assinalaram Certamente Faria ou Provavelmente Faria, revelando um dado preocupante. Apesar de não ser maioria, tal porcentagem foi maior do que no estudo de Runyan et al. (2010), em que 10\% das 813 mães de Embú-SP entrevistadas relataram ter sacudido seus filhos menores de 2 anos de idade no último ano. A diferença entre essas porcentagens pode ser explicada pela metodologia utilizada em ambas as pesquisas. No presente estudo, foi investigada a probabilidade de os pais utilizarem o sacudir como estratégia para acalmar o bebê, enquanto Runyan et al. (2010) investigaram a ocorrência passada de eventos envolvendo o sacudir em bebês. Dadas as implicações negativas de se admitir ter sacudido o próprio filho, é esperado que se encontre um número menor de relatos se comparado a um estudo que investigue apenas a concordância dos pais em utilizar o sacudir para lidar com o choro do bebê.

Outra questão que merece destaque refere-se ao fato de o presente instrumento não deixar exatamente claro no que consiste "sacudir um bebê". Desse modo, é possível que os participantes tenham entendido o termo "sacudir" como balançar ou ninar, estratégias apropriadas que não levam às consequências negativas do sacudir de forma violenta. Ressalta-se, assim, a necessidade de aprimoramento do instrumento, de forma a esclarecer no que consiste o comportamento de sacudir um bebê.

Tabela 3. Porcentagens de resposta para cada item do questionário relativo às estratégias para lidar com o choro do bebê $(n=90)$

\begin{tabular}{|c|c|c|c|}
\hline & $\begin{array}{l}\% \\
\text { Faria }\end{array}$ & $\begin{array}{c}\% \\
\text { Não } \\
\text { Faria }\end{array}$ & $\begin{array}{c}\% \\
\text { Não Sei }\end{array}$ \\
\hline Estratégias Relacionadas à Satisfação das Necessidades Básicas do Bebê & 88,5 & 7,0 & 4,4 \\
\hline Alimentar o bebê novamente & 92,2 & 6,7 & 1,1 \\
\hline Trocar a fralda & 100,0 & 0,0 & 0,0 \\
\hline Trocar a roupa & 73,3 & 14,4 & 12,2 \\
\hline Estratégias Relacionadas ao Bebê & 88,6 & 8,3 & 3,1 \\
\hline Abraçaria o bebê & 95,6 & 3,3 & 1,1 \\
\hline Faria uma massagem no bebê & 94,4 & 4,4 & 1,1 \\
\hline Daria chupeta para o bebê & 62,2 & 7,8 & 30,0 \\
\hline Traria o bebê para perto do corpo & 100,0 & 0,0 & 0,0 \\
\hline Distrairia o bebê com brinquedos & 86,7 & 10,0 & 3,3 \\
\hline Levaria o bebê para um passeio ao ar livre & 87,8 & 4,4 & 7,8 \\
\hline Cantaria uma música para o bebê & 90,0 & 7,8 & 2,2 \\
\hline Ninaria o bebê & 92,2 & 6,7 & 1,1 \\
\hline Estratégias Relacionadas ao Ambiente & 62,6 & 31,1 & 6,3 \\
\hline Colocaria uma música para o bebê & 72,2 & 18,9 & 8,9 \\
\hline Colocaria o bebê em um lugar mais escuro & 20,0 & 71,1 & 8,9 \\
\hline Colocaria o bebê em um lugar mais tranquilo, com menos barulho & 95,6 & 3,3 & 1,1 \\
\hline Estratégias Relacionadas ao Cuidador & 41,7 & 53,3 & 5,0 \\
\hline Colocaria o bebê no berço & 24,4 & 68,9 & 6,7 \\
\hline Deixaria o bebê sozinho no berço e sairia do quarto por um tempo para respirar fundo e se acalmar & 10,0 & 87,8 & 2,2 \\
\hline Consultaria um pediatra ou outro profissional de saúde & 96,7 & 2,2 & 1,1 \\
\hline Pediria para alguém mais experiente cuidar do bebê & 62,2 & 25,6 & 12,2 \\
\hline Pediria para alguém mais calmo cuidar do bebê & 54,4 & 38,9 & 6,7 \\
\hline $\begin{array}{l}\text { Deixaria o bebê sozinho no berço e sairia do quarto por um tempo para assistir televisão ou ouvir uma } \\
\text { música }\end{array}$ & 2,2 & 96,7 & 1,1 \\
\hline Estratégias Negativas & 12,2 & 84,4 & 3,3 \\
\hline Gritaria com o bebê & 1,1 & 98,9 & 0 \\
\hline Daria um tapa na bundinha do bebê & 1,1 & 97,8 & 1,1 \\
\hline Sacudiria o bebê para tentar acalmá-lo & 34,4 & 56,7 & 8,9 \\
\hline
\end{tabular}




\section{Número de Horas Diárias de Choro do Bebê}

Em relação ao conhecimento prévio sobre o padrão de choro do bebê, apenas 32\% dos participantes afirmaram corretamente o número de horas que um bebê normal e saudável pode chorar por dia (de 2 a 3 horas) (Tolliday et. al, 2010). A Figura 1 ilustra a porcentagem de repostas para cada opção dessa questão.

Pode-se observar um desconhecimento dos participantes sobre o número médio de horas que um bebê pode chorar por dia, visto que as porcentagens para cada opção foram

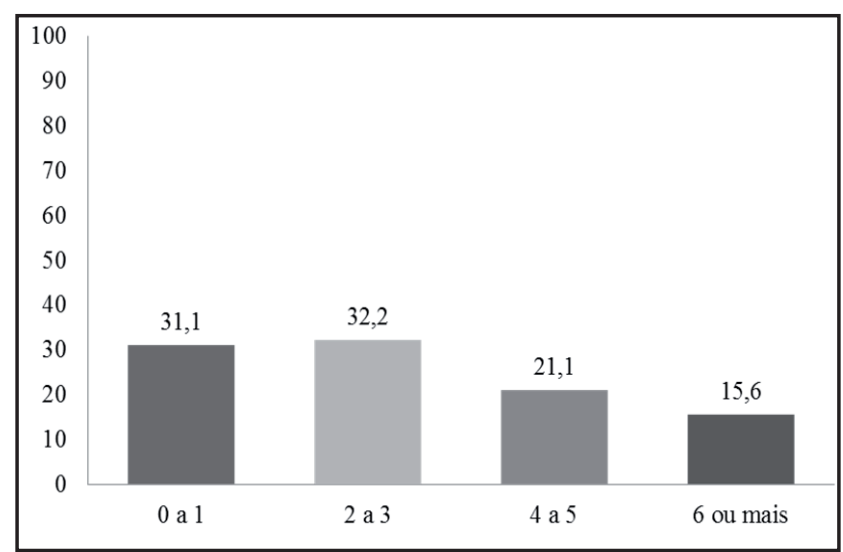

Figura 1. Porcentagem de respostas para cada opção de reposta para a questão sobre o número de horas diário do choro do bebê.

próximas uma das outras. Entretanto, esse desconhecimento parece não ser uma questão apenas desta amostra, visto que, no estudo de Tolliday et al. (2010) na Austrália, 40\% dos participantes relataram corretamente o número médio de horas de choro por dia, porcentagem próxima à do presente estudo.

Esses dados apontam a necessidade de ações educativas dirigidas a pais e outros cuidadores sobre desenvolvimento infantil, de modo que, informados, possam criar expectativas mais realistas sobre o comportamento de seus bebês. No estudo de Tolliday et al. (2010), a exibição de um vídeo informativo sobre TCV aumentou o conhecimento dos participantes sobre o padrão de choro do bebê, indicando algumas possibilidades de intervenções breves que podem vir a ser realizadas no contexto brasileiro.

\section{Sentimentos Associados ao Cuidado do Bebê}

Nash et al. (2008) destacam a importância de se investigarem os sentimentos associados ao choro do bebê, tendo em vista que a ocorrência do TCV tem sido relacionada com sentimentos negativos dos pais quanto ao cuidado com o bebê. No presente estudo, $73 \%$ dos participantes afirmaram que cuidar de um bebê pode ser cansativo e estressante. Esse resultado pode ser considerado relevante no sentido que, ao acreditar que o cuidado de um bebê nem sempre é uma tarefa fácil, pais e cuidadores podem estar mais abertos a programas que os auxiliem a lidar com os próprios sentimentos no cuidado com o filho, bem como solicitar ajuda às redes de apoio.

Diante do fato de $53,3 \%$ dos participantes afirmarem que não utilizariam estratégias relacionadas ao cuidador para lidar com um bebê choroso, destaca-se a necessidade de ensinar a esses pais que a utilização dessas estratégias pode ser adequada e saudável, no sentido de regular suas próprias emoções e prevenir agressões contra o bebê. Sugere-se, entretanto, a inclusão no questionário de outros itens relacionados aos sentimentos evocados no cuidado do bebê. No estudo de Nash et al. (2008), por exemplo, os pais relataram, principalmente, sentirem-se culpados por não serem capazes de acalmar seus bebês. Pensando em possíveis intervenções com pais de recém-nascidos, torna-se relevante a investigação dessas questões no contexto brasileiro.

\section{Consequências de se Sacudir o Bebê}

A Tabela 4 apresenta as porcentagens de resposta para cada item do questionário relativo às consequências de sacudir um bebê, separados por categoria. Observa-se que a categoria que obteve maior concordância foi Consequências Não Graves, com 90\% dos participantes respondendo Sim. Em contraste, a categoria Consequências Inadequadas obteve maior discordância, com $75 \%$ dos participantes respondendo

Tabela 4 Porcentagens de resposta para cada item do questionário relativo às consequências de sacudir um bebê $(n=90)$

\begin{tabular}{|c|c|c|c|}
\hline & $\begin{array}{c}\% \\
\text { Sim }\end{array}$ & $\begin{array}{c}\text { \% } \\
\text { Nâo }\end{array}$ & $\begin{array}{c}\% \\
\text { Não Sei }\end{array}$ \\
\hline Consequências Graves & 50,1 & 15,6 & 34,3 \\
\hline $\begin{array}{l}\text { Futuramente o bebê pode ter } \\
\text { problemas para aprender }\end{array}$ & 62,2 & 13,3 & 24,0 \\
\hline $\begin{array}{l}\text { Futuramente o bebê pode ter } \\
\text { problemas com a fala }\end{array}$ & 48,9 & 13,3 & 38,0 \\
\hline $\begin{array}{l}\text { O bebê pode desenvolver deficiência } \\
\text { física }\end{array}$ & 55,6 & 11,1 & 33,0 \\
\hline $\begin{array}{l}\text { O bebê pode desenvolver deficiência } \\
\text { mental }\end{array}$ & 52,2 & 15,6 & 32,0 \\
\hline $\begin{array}{l}\text { O bebê pode desenvolver problemas } \\
\text { com a visão }\end{array}$ & 31,1 & 24,4 & 43,0 \\
\hline O bebê pode ter paralisia cerebral & 38,9 & 20,0 & 41,0 \\
\hline O bebê pode ter parada cardíaca & 33,3 & 21,1 & 46,0 \\
\hline O bebê pode ter convulsões & 63,3 & 6,7 & 30,0 \\
\hline Pode levar à morte do bebê & 65,6 & 13,3 & 21,0 \\
\hline Consequências Não Graves & 90 & 2,5 & 7,5 \\
\hline O bebê pode vomitar & 100,0 & 0,0 & 0,0 \\
\hline $\begin{array}{l}\text { O bebê pode não conseguir se } \\
\text { alimentar }\end{array}$ & 77,8 & 3,3 & 19,0 \\
\hline O bebê pode ficar mais nervoso & 90,0 & 4,4 & 6,0 \\
\hline O bebê pode chorar mais & 93,3 & 2,2 & 4,0 \\
\hline Consequências Inadequadas & 15,6 & 75 & 9,4 \\
\hline Nada & 7,8 & 80,0 & 12,0 \\
\hline O bebê pode se acalmar & 23,3 & 70,0 & 7,0 \\
\hline
\end{tabular}


"Não". Esses dados parecem indicar que os participantes do estudo acreditam que sacudir um bebê pode trazer consequências negativas a ele, sendo as mais frequentemente apontadas: vômitos (100\%), ficar ainda mais nervoso (90\%), chorar ainda mais $(93,3 \%)$ e dificuldade para se alimentar $(77,8 \%)$.

Pode-se observar, entretanto, que $34,4 \%$ dos participantes responderam Não Sei para os itens da categoria Consequências Graves, indicando que, apesar de acreditarem que sacudir um bebê leva a consequências negativas, parecem não saber se as mesmas podem ser graves. Nesse sentido, os resultados apontam para uma falta de conhecimento sobre os efeitos nocivos dessa forma de violência para suas vítimas. Esses resultados são similares aos encontrados por Tolliday et al. (2010), segundo os quais apenas $17 \%$ dos participantes acreditavam que sacudir um bebê poderia levar à morte.

Observa-se, ainda, que $23,3 \%$ dos participantes afirmaram que sacudir o bebê pode ajudar a acalmá-lo. Considerando esse resultado em conjunto com o dado anterior de que $34,4 \%$ sacudiriam seu bebê para tentar acalmá-lo, pode-se inferir que existe uma amostra expressiva de participantes, ainda que não a maioria, que acredita que sacudir o bebê pode trazer benefícios, aumentando a probabilidade de fazê-lo. Cabe destacar, novamente, que a porcentagem do presente estudo pode estar inflada pelo entendimento do sacudir como balançar ou ninar, e não como um comportamento violento.

Cabe ressaltar, entretanto, que essa porcentagem é consideravelmente menor do que a encontrada no estudo de Tolliday et al. (2010), na Austrália, em que 64\% dos participantes acreditavam que sacudir um bebê poderia acalmá-lo. Esses resultados apontam, novamente, para uma possível diferença cultural em relação às crenças e práticas relacionadas ao cuidado do bebê. Essa diferença evidencia a necessidade de estudos nacionais sobre o tema, de modo que as intervenções para prevenção do TCV estejam mais adequadas para o contexto brasileiro.

\section{Conclusão}

O presente estudo sugere um desconhecimento por parte dos pais e cuidadores sobre o TCV, principalmente no que refere à sua gravidade. Além disso, o estudo aponta para uma possível crença de que o choro do bebê é ocasionado por seus traços pessoais, o que prejudica as possíveis intervenções nessas situações. Ressalta-se, entretanto, que esses resultados não podem ser ampliados para a população brasileira, devido ao tamanho reduzido da amostra, servindo como dados inicias a serem confirmados em pesquisas posteriores.

Outra limitação do presente estudo refere-se à utilização de um questionário adaptado, cuja construção parece não ter sido orientada de acordo com as boas práticas em psicometria (Ziegler, 2014). Nesse sentido, o instrumento não contempla todas as dimensões relacionadas à prevenção do $\mathrm{TCV}$, como por exemplo, o conhecimento parental sobre o padrão de choro nos primeiros meses de vida, além de apresentar um número assimétrico de itens para cada dimensão investigada. Tais limitações apontam para a necessidade de elaboração de novos instrumentos que avaliem todas as dimensões envolvidas com a ocorrência do TCV e cujas propriedades psicométricas sejam adequadamente investigadas.

Ao considerar os resultados do presente estudo em conjunto com os de Fernandes et al. (2010), em que 97\% dos profissionais de saúde entrevistados não conheciam o $\mathrm{TCV}$, parece haver um real desconhecimento sobre o TCV no Brasil. Vale ressaltar que os estudos foram realizados em regiões diferentes do país, indicando que talvez esse desconhecimento não seja característica regional.

Em estudo realizado por Runyan et al. (2010) em município de médio porte do estado de São Paulo, $10 \%$ das mães relataram já ter sacudido seus filhos no último ano quando estes tinham menos de 2 anos de idade (Runyan et. al., 2010). Observa-se, assim, que o aparente desconhecimento do TCV no Brasil não parece significar a inexistência dessa forma de maus-tratos no país. A principal implicação desse desconhecimento é a ausência de intervenções e programas específicos para a prevenção de casos de TCV. Ressalta-se, assim, a importância de se realizarem novas pesquisas sobre a temática, investigando o conhecimento de cuidadores e profissionais sobre o TCV e a prevalência desta forma de maus-tratos no Brasil e avaliando estratégias para sua prevenção.

\section{Referências}

Alvarez, M., \& James-Roberts, I. S. (1996). Infant fussing and crying patterns in the first year in an urban community in Denmark. Acta Paediatrica, 85(4), 463-466.

Barr, R. G. (2001). "Colic" is something infants do, rather than a condition they "have": A developmental approach to crying phenomena, patterns pacification and (patho)genesis. In R. G. Barr, I. S. James-Roberts, \& M. R. Keefe (Eds.), New evidence on unexplained early infant crying: Its origins, nature and management (pp. 87-104). Miami, FL: Johnson \& Johnson Pediatric Institute.

Barr, R. G. (2014). Crying as a trigger for abusive head trauma: A key to prevention. Pediatric Radiology, 44(Suppl. 4), S559-S564.

Barr, R. G., Chen, S., Hopkins, B., \& Westra, T. (1996). Crying patterns in preterm infants. Developmental Medicine and Child Neurology, 38(4), 345-355.

Barr, R. G., Konner, M., Bakeman, R., \& Adamson, L. (1991). Crying in !Kung San infants: A test of the cultural specificity hypothesis. Developmental Medicine and Child Neurology, 33(7), 601-610.

Barr, R. G., Paterson, J. A., MacMartin, L. M., Lehtonen, L., \& Young, S. N. (2005). Prolonged and unsoothable crying bouts in infants with and without colic. Developmental and Behavioral Pediatrics, 26(1), 14-23.

Barr, R. G., Trent, R. B., \& Cross, J. (2006). Age-related incidence curve of hospitalized Shaken Baby Syndrome cases: Convergent evidence for crying as a trigger to shaking. Child Abuse \& Neglect, 30(1), 7-16.

Brazelton, T. B. (1962). Crying in infancy. Pediatrics, 29, 579-588. 
Brooks, W., \& Weathers, L. (2001). Overview of Shaken Baby Syndrome. In S. Lazoritz \& V. J. Palusci (Eds), The Shaken Baby Syndrome: A multidisciplinary approach (pp. 1-8). Binghamton, NY: The Haworth Maltreatment \& Trauma Press.

Case, M. E., Graham, M. A., Handy, T. C., Jentzen, J. M., \& Monteleone, J. A. (2001). Position paper on fatal abusive head injuries in infants and young children. The American Journal of Forensic Medicine and Pathology, 22(2), 112-122.

Center of Disease and Control. (2007). Child Maltreatment: Factsheet. Acesso em 13 de Julho de 2010, disponível em http://www.cdc.gov/ncipc/factsheets/cmfacts.htm

Commitee on Child Abuse and Neglect of the American Academy of Pediatrics. (2001). Shaken Baby Syndrome: Rotational cranial injuries - tecnical report. Pediatrics, 108(1), 206-210.

Cozby, P. C. (2011). Métodos de pesquisa em ciências do comportamento. São Paulo, SP: Editora Atlas.

Deyo, G., Skybo, T., \& Carroll, A. (2008). Secondary analysis of the "Love Me...Never Shake Me" SBS education program. Child Abuse \& Neglect, 32, 1017-1025.

Dias, M. S., Smith, K., DeGuehery, K., Mazur, P., Li, V., \& Shaffer, M. L. (2005). Preventing Abusive Head trauma among infants and young children: A hospital-based, parent education program. Pediatrics, 115(4), 470-477.

Dix, T., \& Reinhold, D. P. (1991). Chronic and temporary influences on mothers' attributions for children's desobedience. MerrillPalmer Quarterly, 37(2), 251-271.

Fernandes, V. M., Silva, N. L., \& Javorski, M. (2010). Prevenção da Síndrome do Bebê Sacudido: Conhecimento da equipe de Saúde da Família. Nursing, 13(145), 304-308.

Frasier, L. D. (2008). Abusive head trauma in infants and young children: A unique contributor to developmental disabilities. Pediatric Clinics of North America, 55(6), 1269-1285.

Fujiwara, T., Yamada, F., Okuyama, M., Kamimaki, I., Shikoro, N., \& Barr, R. G. (2012). Effectiveness of educational materials designed to change knowledge and behavior about crying and shaken baby syndrome: A replication of a randomized controlled trial in Japan. Child Abuse \& Neglect, 36(9), 613620.

Green, J. A., \& Gustafson, G. E. (2001). Crying: Multiple determinants of perceived meaning. In R. G. Barr, I. S. JamesRoberts, \& M. R. Keefe (Eds.), New evidence on unexplained early infant crying: Its origins, nature and management (pp. 105-120). Miami, FL: Johnson \& Johnson Pediatric Institute.

James-Roberts, I. S. (2001). Infant crying and its impact on parents. In R. G. Barr, I. S. James-Roberts, \& M. R. Keefe (Eds.), New evidence on unexplained early infant crying: Its origins, nature and management (pp. 5-24). Miami, FL: Johnson \& Johnson Pediatric Institute.

James-Roberts, I. S. \& Halil, T. (1991). Infant crying patterns in the first year: Normal community and clinical findings. Journal of Child Psychology and Psychiatry, 32(6), 951-968.

Health Canada. (2001). Joint Statement on Shaken Baby Syndrome. Ottawa: Minister of Public Works and Government Services.

Hennes, H., Kini, N., \& Palusci, V. J. (2001). The epidemiology, clinical characteristcs and public health implications of Shaken Baby Syndrome. In S. Lazoritz \& V. J. Palusci (Eds.), The Shaken Baby Syndrome: A multidisciplinary approach (pp. 19-40). Binghamton, NY: The Hayworth Maltreatment \& trauma Press.
Hofer, M. A. (2001). Infant crying: An evolutionary perspective. In R. G. Barr, I. S. James-Roberts, \& M. R. Keefe (Eds.), New evidence on unexplained early infant crying: Its origins, nature and management (pp. 59-70). Miami, FL: Johnson \& Johnson Pediatric Institute.

Hunziker, U. A., \& Barr, R. G. (1986). Increased carrying reduces infant crying: A randomized controlled trial. Pediatrics, 77(5), 641-648.

Keenan, H. T., \& Leventhal, J. M. (2010). A Case-Control Study to Evaluate Utah's Shaken Baby Prevention Program. Academic Pediatrics, 10(6), 389-394.

Kramer, M. S., Barr, R. G., Dagenais, S., Yang, H., Jones, P., Ciofoni, L., \& Jané, F. (2001). Pacifier use, early weaning, and cry/fuss behavior: A randomized controlled trial. The Journal of the American Medical Association, 286(3), 322-326.

Leavitt, L. A. (2001). Infant crying: Expectations and parental response. In R. G. Barr, I. S. James-Roberts, \& M. R. Keefe (Eds.), New evidence on unexplained early infant crying: Its origins, nature and management (pp. 43-50). Miami, FL: Johnson \& Johnson Pediatric Institute.

Lee, C., Barr, R. G., Catherine, N., \& Wicks, A. (2007). Age-related incidence of publicly reported Shaken Baby Syndrome cases: Is crying a trigger for shaking? Journal of Developmental and Behavioral Pediatrics, 28(4), 288-293.

Lopez, F. A. \& Júnior, D. C. (2010). Filhos: Da gravidez aos 2 anos de vida; dos pediatras da Sociedade Brasileira de Pediatria para os pais. Barueri, SP: Manole.

Nash, C., Morris, J., \& Goodman, B. (2008). A study describing mothers' opinion of the crying behavior of infants under one year of age. Child Abuse Review, 17, 191-200.

National Center on Shaken Baby Syndrome. (s.d.). What does science tell us about abusive head trauma in infants and young children? Acesso em 13 de Julho de 2010, disponível em http://www.dontshake.org/sbs.php?topNavID=3\&subNav $\mathrm{ID}=25$ \&navID $=280$.

Parks, S.E., Annest, J. L., Hill, H. A., \& Karch, D. L. (2012). Pediatric abusive head trauma: Recommended definitions for public health surveillance and research. Atlanta, GA: Centers for Disease Control and Prevention.

Pettijohn, T. F. (1979). Attachment and separation distress in the infant guinea pig. Developmental psychobiology, 12(1), 73-81.

Reijneveld, S. A., Wal, M. F. D., Brugmanm, E., Sing, R. A. H., \& Verloove-Vanhorick, S. P. (2004). Infant crying and abuse. Lancet, 364(9442), 1340-1342.

Runyan, D. K., Shankar, V., Hassan, F., Hunter, W. M., Jaind, D., Paula, ... Bordin, I. A. (2010). International variations in harsh child discipline. Pediatrics, 126(3), e701-e711.

Runyan, D. K., Hennink-Kaminski, H. J., Zolotor, A. J., Barr, R. G., Murphy, R. A., Barr, M., ... Nocera, M.. (2009). Designing and Testing a Shaken Baby Syndrome Prevention Program The Period of PURPLE Crying: Keeping babies safe in North Carolina. Social Marketing Quarterly, 15(4), 2-24.

Runyan, D. K., \& Zolotor, A. (2009). The Period of PURPLE Crying: Keeping babies safe in North Carolina. In K. A. Dodge \& D. L. Coleman (Eds.), Preventing Child Maltreatment Community Approaches (pp. 102-118). New York, NY: The Guilford Press.

Russell, B. S. (2010). Revisiting the measurement of Shaken Baby Syndrome Awareness. Child Abuse \& Neglect, 34(9), 671-676. 
Sisto, F. F. (2004). Traços de personalidade de crianças e emoções: Evidência de validade. Paidéia (Ribeirão Preto), 14(29), 359-369.

Tolliday, F., Simons, M., Foley, S., Benson, S., Stephens, A., \& Rose, D. (2010). From inspiration to action: The Shaken Baby Prevention Project in Western Sydney. Communities, Children and Families in Australia, 5(2), 31-47.
Wessel, M. A., Cobb, J. C., Jackson, E. B., Harris, G. S., \& Detwiler, A. C. (1954). Paroxysmal fussing in infant, sometimes called "colic". Pediatrics, 14(5), 421-434.

Ziegler, M. (2014). Stop and state your intentions! Let's not forget the $\mathrm{ABC}$ of test construction. European Journal of Psychological Assessment, 30(4), 239-24

Recebido em 31.01.2013

Primeira decisão editorial em 24.08.2015

Versão final em 11.11.2015

Aceito em 09.02.2016 